\title{
Lithium in drinking water and suicide mortality: interplay with lithium prescriptions
}

Marco Helbich, Michael Leitner and Nestor D. Kapusta

\section{Background}

Little is known about the effects of lithium intake through drinking water on suicide. This intake originates either from natural rock and soil elution and/or accumulation of lithium-based pharmaceuticals in ground water.

\section{Aims \\ To examine the interplay between natural lithium in drinking water, prescribed lithium-based pharmaceuticals and suicide in Austria.}

\section{Method}

Spatial Bayesian regressions for males, females and pooled suicide mortality rates were estimated.

\section{Results}

Although the expected inverse association between lithium levels in drinking water and suicide mortality was confirmed for males and for total suicide rates, the relationship for females was not significant. The models do not indicate that lithium from prescriptions, assumed to accumulate in drinking water, is related to suicide risk patterns either as an individual effect or as a moderator of lithium levels in drinking water. Gender-specific differences in risk factors and local risk hot spots are confirmed.

\section{Conclusions}

The findings do not support the hypotheses that lithium prescriptions have measureable protective effects on suicide or that they interact with lithium in drinking water.

\section{Declaration of interest}

None.

\section{Copyright and usage}

(c) The Royal College of Psychiatrists 2015. This is an open access article distributed under the terms of the Creative Commons Non-Commercial No Derivatives (CC BY-NC-ND) licence.
Suicide is one of the largest health burdens in industrialised nations. ${ }^{1}$ The Organisation for Economic Co-operation and Development ${ }^{2}$ reports that suicide mortality rates in Austria are constantly above the European average. In the period 2005-2009 the mean Austrian suicide rate was 15.7 per 100000 people showing a distinctive gender gap with a suicide rate ratio of 3.3:1 in favour of women. Suicide is a complex phenomenon affected by a variety of interwoven risk and protective factors (i.e. sociodemographic characteristics, access to health services and environmental factors). ${ }^{3,4}$ The complexity of suicide epidemiology is amplified because of considerable risk variations between nations and within countries. ${ }^{2,5,6,7}$ Mental disorders, especially depression and bipolar disorders, are well-known risk factors for suicide, increasing mortality by up to 20 times. ${ }^{8}$ Because of its mood-stabilising effects and suicide preventive properties, lithium is used for acute and prophylactic treatments in psychiatry. ${ }^{9,10}$ Meta-analyses of clinical trials have found that suicide risk among people affected by mood disorders is significantly reduced when they are treated with lithium. ${ }^{11,12}$ A cohort study in Denmark concluded that lithium treatment diminishes suicide risk independently of a person's age and gender, even though the suicide risk is approximately 10 times higher for patients taking lithium than the general population. ${ }^{13}$ However, whereas the effects of therapeutic dosages of lithium are well understood there is a lack of knowledge on the effects of natural lithium intake.

Lithium, as a natural trace element, is eluded from rock and soil and accumulates in groundwater. These natural doses are significantly lower than those used in therapeutic treatment. ${ }^{14}$ It is assumed that long-term exposure to even minor lithium levels might act as a suicide preventive. However, scientific evidence is far from conclusive and contradictory empirical results have been reported for different regions. Among one of the first studies reporting a negative effect of natural lithium in drinking water on suicide was conducted in Texas, USA. ${ }^{14}$ After adjusting for risk factors, a more recent study in this area confirmed that lithium in public water is negatively but weakly related to suicide mortality. ${ }^{15}$ In the Oita prefecture in Japan, lithium levels in tap water were found to diminish suicide risk significantly, ${ }^{16}$ even though methodological flaws have been highlighted. ${ }^{17,18}$ Sugawara et $a l^{19}$ reported gender differences with only a significant negative association between lithium in drinking water and suicide mortality among Japanese women. These studies sparked interest in Europe. For the East of England and prefectures in Greece neither significant associations between suicide and lithium in public water supply nor gender differences were found. ${ }^{20,21}$ In contrast, in Austria the natural lithium-suicide association was confirmed, although statistical significance was limited to eastern regions. ${ }^{5}$

Previous studies have assumed that the lithium in drinking water originated from natural sources alone (the natural lithium hypothesis). They have not considered whether lithium prescribed to patients may have accumulate via waste water in groundwater aquifers after urinary excretion and interplay with natural lithium. ${ }^{22}$ Strong evidence exists that pharmaceuticals in waste water give rise to public health problems such as antibiotics causing resistant bacteria in waste water. ${ }^{23,24}$ Pharmaceuticals, such as carbamazepine and ibuprofen, and also biphosphenol A have been detected in Canadian drinking water, ${ }^{25}$ and acetaminophen caffeine, carbamazepine, codeine have been found in drinking water in California. ${ }^{26}$ There is some evidence that the regions with high prescriptions of pharmaceuticals, such as in Los Angeles, have higher levels of pharmaceuticals in groundwater than other regions in California. ${ }^{26}$ In the USA, levels of the common antidepressant fluoxetine were measurable in $4.3 \%$ of all investigated groundwater sites. ${ }^{27}$ Nentwig $^{28}$ confirmed that the effects of fluoxetine and carbamazepine concentrations in sediment and water systems were strong enough to affect health. Therefore, it is assumed that lithium may accumulate in drinking water reservoirs and modify its natural occurrence and thus may be 
responsible for a non-random distribution of lithium in drinking water across regions. Although the findings discussed above have contributed significantly to our knowledge about the epidemiology of suicide, no research known to the authors deals with the relationship between prescribed lithium-based pharmaceuticals, natural lithium levels in drinking water and suicide mortality. In addition, gender differences are rarely considered separately, as higher and lower suicide rates tend to cluster spatially. Our study addresses these research gaps and focuses on the following research questions: (a) are lithium levels in drinking water associated with lithium prescriptions; (b) is there statistical evidence that any association found between suicide and lithium levels in drinking water is a function of lithium prescription rates operating as a moderating effect; and (c) are there gender-specific variations concerning both lithium-based variables (i.e. drinking water and lithium prescriptions) while controlling for other risk factors? To examine whether the natural lithium hypothesis holds true or whether the lithium levels in drinking water may result from the accumulation of lithium prescriptions in waste water (lithium prescription hypothesis), we generated the following hypotheses. First, districts with a high lithium level in drinking water also have high lithium prescription rates and both lithium sources are inversely associated with suicide rates, even when risk and protective factors are adjusted for. Second, natural lithium levels in drinking water have a suicide protective effect, even when adjusted for lithium prescriptions. Besides being vital for health prevention strategies, testing the lithium prescription hypothesis is of importance to medicine, public health and environmental science.

\section{Method}

\section{Study area}

The empirical analysis was conducted in Austria using an ecological study design. Austria represents an excellent case study to explore the potential interaction between prescriptions rates and lithium in drinking water on suicide mortality. This is because of (a) its unique topography leading to different geological bedrocks and thus different natural lithium levels, (b) its alpine areas with reduced health service accessibility and (c) knowledge about the existence of essential confounding risk factors, therefore reducing the risk of an omitted variable bias. The study is based on 99 administrative territorial units (districts) from which suicide cases were available. In order to link the suicide numbers with prescription data available for each sanitation district, a few administrative districts were carefully aggregated to match the sanitation districts $(n=90)$.

\section{Data}

The suicide data were collected from the Austrian mortality database, maintained by Statistics Austria (http://www.statistik.at/web_en/), on a case level. Suicide data comprise all registered suicides across all ages (with the youngest in the 5-9 age group and the oldest in the $95+$ age group) within the 5-year period from 2005 to 2009. In accordance with ICD- $10^{29}$ the data include the following causes of death: poisoning (X60-X69), hanging (X70), drowning (X71), shooting (X72-X74), jumping (X80) and other less frequent causes of death $(\mathrm{X} 75-\mathrm{X} 79, \mathrm{X} 81-\mathrm{X} 84)$. As suicide mortality is a rare event, the annual data were pooled over time to reduce the random fluctuations of suicides within a district. Moreover, because the raw number of suicides per district depends on the underlying population at risk, age and gender-adjusted standardised suicide mortality ratios (SMRs) were calculated. On average, the gender ratio in Austria is approximately 51\% women and 49\% men. The population counts extracted from the census of 2001 (Statistics Austria) serve as a baseline. The SMRs reflect the ratio
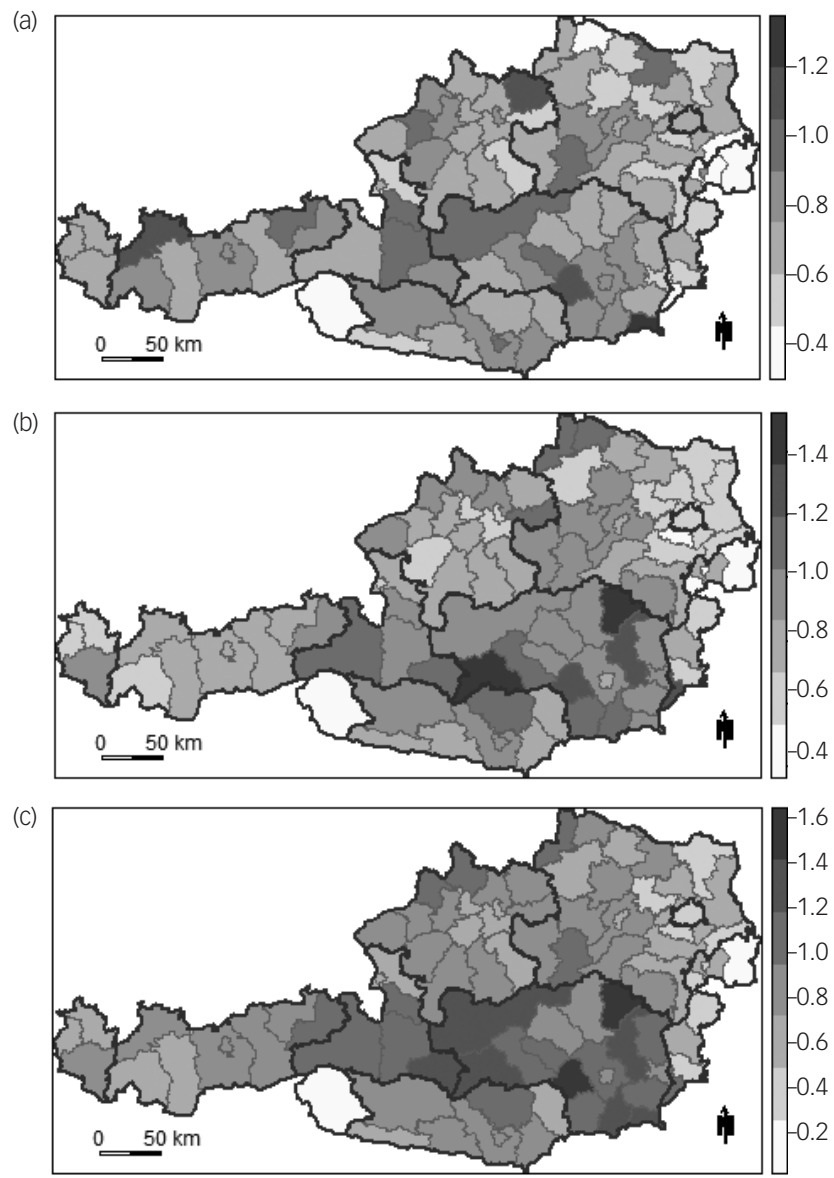

Fig. 1 Spatial distribution of the total and gender-specific suicide rates.

Suicide mortality ratios (a) total, (b) males and (c) females. Dark grey lines delimit federal states; light grey lines define sanitation district boundaries.

between observed and expected suicides within each district. Mapping of the total SMR $\left(\mathrm{SMR}_{\mathrm{t}}\right)$ and gender-specific SMRs for males and females $\left(\mathrm{SMR}_{\mathrm{m}}, \mathrm{SMR}_{\mathrm{f}}\right.$, respectively) in Fig. 1 indicate spatial heterogeneity in risk. Southern areas are faced with a more distinct risk compared with northeastern areas.

Besides suicide data, two other data-sets of prime interest were obtained, namely the lithium content in drinking water and lithium pharmaceuticals sales. Data about the former were provided by AQA GmbH (http://www.aqa-online.com) and came from 6460 water samples from across Austria. ${ }^{3}$ To merge the individual lithium measurements with the district-level data, the mean lithium concentration per district was computed and log transformed to a Gaussian-like distribution (logLITH). On average the lithium content was $0.010 \mathrm{mg} / \mathrm{L}$ with a standard deviation of $0.010 \mathrm{mg} / \mathrm{L}$. The lowest lithium level was $0.003 \mathrm{mg} / \mathrm{L}$, which corresponds to the minimum measurement accuracy of the spectrometry. Figure 2(a) shows a geographically clear spatial variation, following an east-west trend. Eastern regions have higher lithium levels $(>0.016 \mathrm{mg} / \mathrm{l})$ than western parts $(0.004 \mathrm{mg} / \mathrm{l})$.

Data on lithium pharmaceuticals sales in pharmacies were obtained from IMS Health (http://www.goeg.at/en). The number of prescriptions were based on sold units (i.e. packages, tablets) for the two lithium-based medical products, namely Quilonorm and Neurolepsin (lithium-carbonate), used in psychiatric treatment in 2010 and 2011 and these were converted to the daily defined dose (DDD). As depicted in Fig. 2(b), logged lithium 

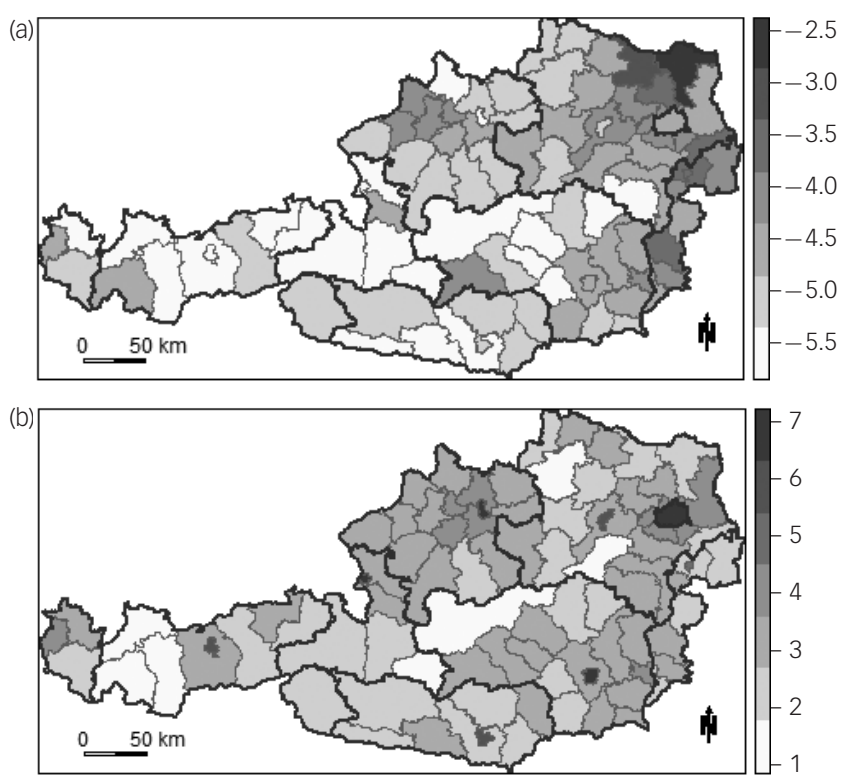

Fig. 2 Spatial distribution of lithium-based variables

(a) Logged natural lithium in drinking water and (b) logged lithium prescriptions. Dark grey lines delimit federal states; light grey lines define sanitation district boundaries.

DDDs sold per $\mathrm{km}^{2}$ (logPHARM) increased with urbanisation and is exceptionally high in the (provincial) capital cities including Vienna (1360.2 DDD per $\mathrm{km}^{2}$ ), Graz (1286.2 DDD per $\mathrm{km}^{2}$ ) and Linz (894.8DDD per $\mathrm{km}^{2}$ ). The nationwide average is approximately 85 DDD per $\mathrm{km}^{2}$ with a standard deviation of 85.2.

The selection of the considered controls was based upon data availability and suggestions from the literature. ${ }^{4}$ To account for known risk factors, the most complete data on the proportion of Roman Catholics (ROMCAT), population density (POPDENS) and average income per capita (INCOME) were obtained from Statistics Austria for the year 2001. Additional data on the density of psychiatrists per 10000 population for the year 2007 (PSYC), the number of general practitioners for 2007 (GP) (both provided by the Austrian Medical Chamber), and the density of psychotherapists per 10000 people for the year 2005 (PT) were obtained from the Austrian Institute of Health to account for possible protective effects of the local psychosocial services. Average unemployment rates for the period 2005-2008 per district (UNEMPL) were collected from the Austrian Public Employment Service. Finally, in order to take spatial heterogeneity in suicide risks into account, ${ }^{5}$ the 99 districts from nine federal states were aggregated into four larger regions (west - Vorarlberg, Tyrol; south - Carinthia, Styria; central - Salzburg, Upper Austria; and east - Lower Austria, Vienna, Burgenland) and considered as regional dummy variables (REG) in the analysis.

\section{Statistical analysis}

Conditional plots were utilised to explore the associations between the two lithium-based variables conditioned on certain ranges of the SMRs. Because of the exceptionally high lithium prescription densities in capital cities, as well as peculiarities within their water supplies separate conditional plots with and without capital cities were drawn. Supportive bivariate Spearman's $\rho$ correlation coefficients were calculated to investigate the nature and magnitude of associations between variables. As indicated by prior studies, ${ }^{6,7}$ SMRs do not exhibit a spatially random distribution, which has serious consequences for multivariate non-spatial analysis (i.e. ordinary least squares regression) assuming that observations are independent of each other. To quantify the extent to which a district value is similar to the adjacent district values, Moran's $I$ statistic was calculated. ${ }^{5}$ Positive values smaller or equal to +1 refer to positive spatial autocorrelation (i.e. similar values are spatially nearby), whereas negative values larger or equal to -1 indicate negative spatial autocorrelation (i.e. dissimilar values are nearby). A Moran's I statistic of slightly below 0 confirms spatial independence. Significance was tested based on pseudo $P$-values obtained from 9999 permutations against the null hypothesis of spatial randomness providing the foundation for spatial regression modelling.

To control for potential confounding factors, multivariate spatial Bayesian hierarchical models were implemented. ${ }^{6}$ This suite of regression models provides a powerful approach to model spatial autocorrelation based on unmodelled risk effects, actual spatial patters in SMRs, etc. Spatial correlations were accounted for at a second hierarchical level by means of spatial random effects, such as the conditional autoregressive (CAR) prior distribution. Supported by a simulation study, ${ }^{30}$ this research applies CAR priors after Leroux (for a discussion see Lee ${ }^{30}$ ) permitting locally differentiated smoothing. On that basis, the random effects were set up with independent and diffuse Gaussian priors and the model estimation utilised Markov chain Monte Carlo (MCMC) simulations. As the number of confounders was large, some may feature multicollinearity. Since the impact of individual confounders is of secondary interests, principal component analysis (PCA) was employed to obtain a reduced set of latent variables (principal components) that are uncorrelated and depict the overall risk factor variability.

\section{Results}

Bivariate relationships between total and gender-specific SMRs, as well as $\operatorname{logLITH}$ and $\log$ PHARM were investigated using Spearman correlations (Table 1). LogLITH is, as expected, consistently negatively related with all three SMRs. Similarly, with the exception of $\mathrm{SMR}_{\mathrm{f}}, \log \mathrm{PHARM}$ is statistically significantly negatively associated with $\mathrm{SMR}_{\mathrm{t}}$ and $\mathrm{SMR}_{\mathrm{m}}$ at least at the 0.05 level, meaning that areas with high prescription rates face lower

\begin{tabular}{|c|c|c|c|}
\hline Dependent variable & Independent variable & Spearman's $\rho$ & $P$ \\
\hline SMR, total & $\log L I T H$ & -0.370 & $<0.001$ \\
\hline SMR, total & logPHARM & -0.290 & 0.005 \\
\hline SMR, males & logLITH & -0.320 & 0.003 \\
\hline SMR, males & logPHARM & -0.270 & 0.010 \\
\hline SMR, females & IogLITH & -0.280 & 0.009 \\
\hline SMR, females & IogPHARM & -0.040 & 0.674 \\
\hline $\log L I T H$ & logPHARM & 0.190 & 0.076 \\
\hline
\end{tabular}


suicide mortality. Moreover, there is a tendency $(P<0.100)$ that $\log$ LITH is positively associated with $\log$ PHARM.

To further explore the association between the lithium-based variables for different ranges of SMRs, conditional plots are shown in Fig. 3. Capital cities have high lithium prescription densities and function as leverage points dominating the regression line. In general, scatter plots indicate that $\operatorname{logLITH}$ and $\log$ PHARM were positively associated across different ranges of SMRs and gender. As logPHARM is much more pronounced in cities and thus distorts associations, it is mandatory to control for that issue. In this regard, population density seems to be a rational proxy variable. Figure 3(b), (d) and (f) show a slight tendency that in higher suicide risk areas the $\operatorname{logLITH-} \operatorname{logPHARM}$ association is more positive than in lower risk areas. However, focusing on a specific SMR range reduces the sample size and results should be interpreted with caution.

Furthermore, to evaluate the spatial patterns of each variable, the Moran's I statistic with the five nearest neighbours were

(a)
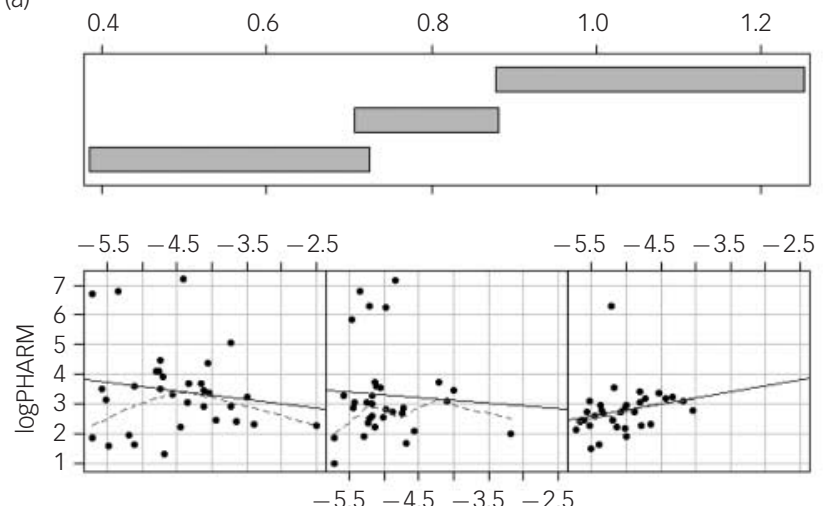

0.6

0.8

1.0

1.2

1.4
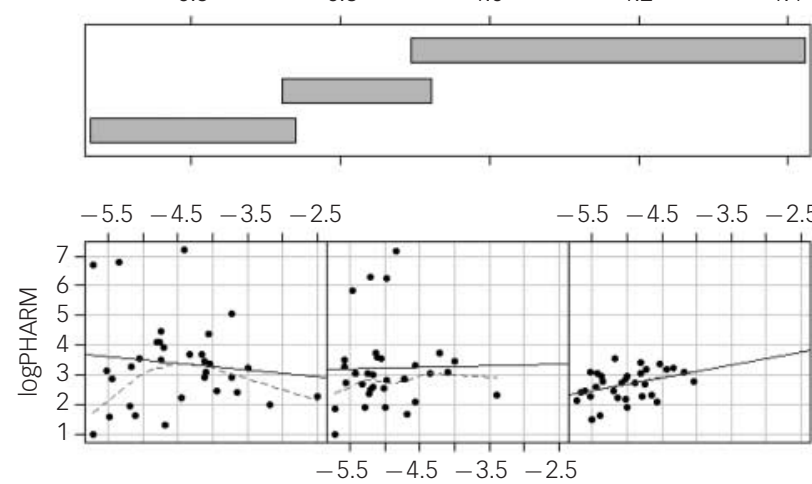

(e)
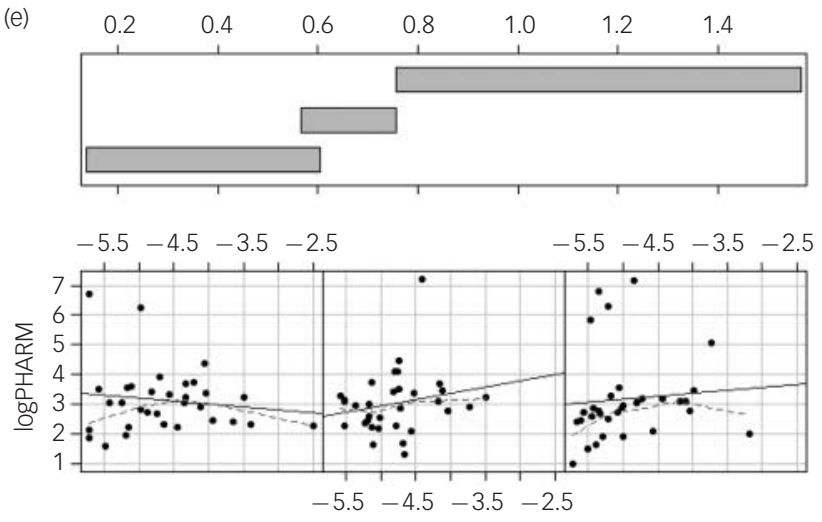

investigated ensuring that each district has a similar number of neighbours. Table 2 lists the results. The Moran's I statistic is highly significant for $\mathrm{SMR}_{\mathrm{t}}$ and the $\mathrm{SMR}_{\mathrm{m}}$, indicating that similar values are located nearby. This finding is critical for subsequent analysis calling for a spatially explicit regression. Furthermore, Spearman correlations point to multicollinearity between some continuous risk factors requiring PCA to establish some uncorrelated principal components. The first three principal components, explaining in total approximately $84 \%$ of the overall variance, were selected for further analysis. Principal component (PC) 1 explains $58 \%$ of the variance, PC2 15\%, and PC3 11\%. In brief, the variables $\log$ POPDENS, $\log$ INCOME, $\log \mathrm{PSYC}, \log \mathrm{PT}$ and logGP load moderate negatively on PC1, whereas logUNEMPL loads lower negatively. In contrast, logROMCAT has a positive loading. LogUNEMPL possesses a negative loading on PC2, whereas other variables show minor positive or negative loadings. PC3 is mainly shaped by $\log \mathrm{GP}$ and $\operatorname{logROMCAT}$, which both having a strong positive loading. Even though more difficult to interpret, these

(b)
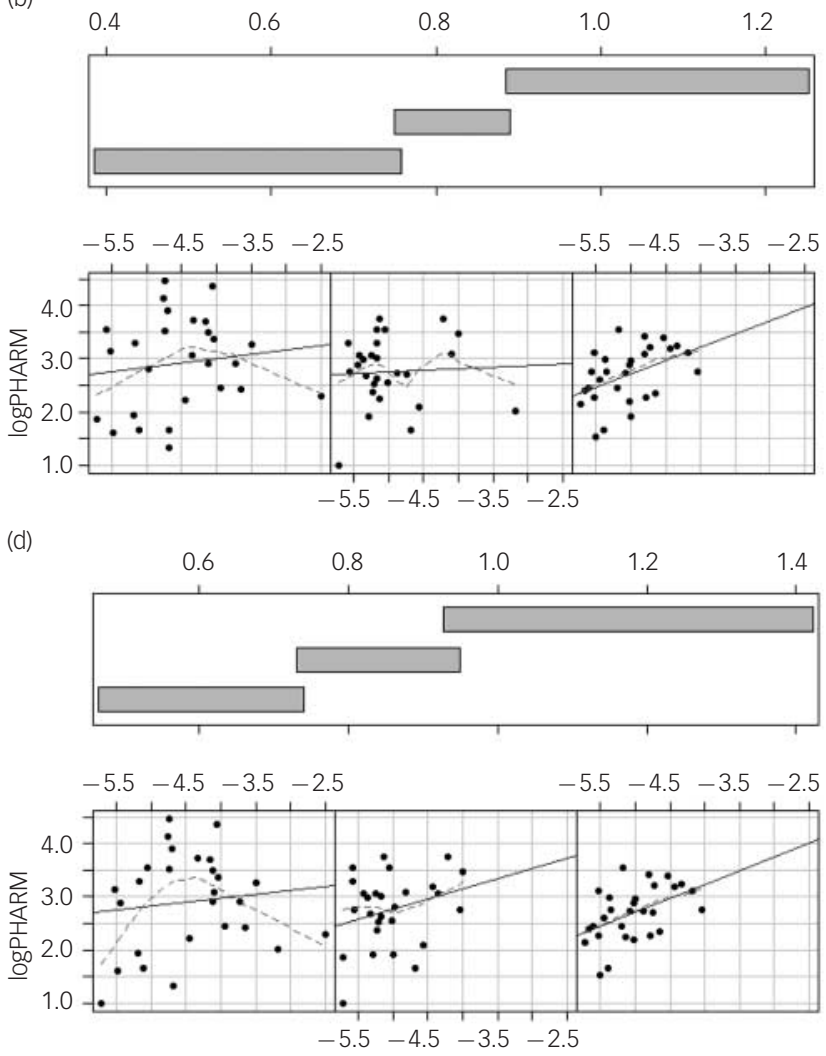

$(f)$
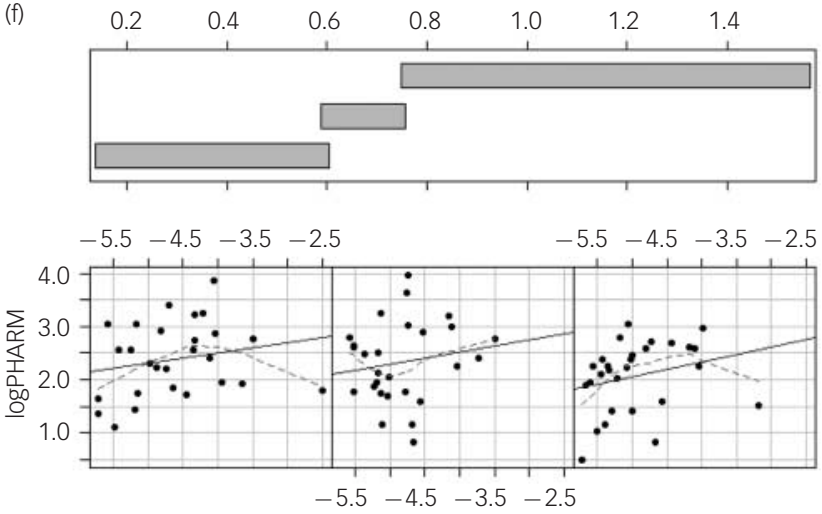

Fig. 3 Conditional plots.

Solid lines refer to a linear regression and dotted lines to a locally weighted polynomial regression. Suicide mortality ratios (a) total, (b) total, without capital cities; (c) males, (d) males, without capital cities; (e) females and (f) females, without capital cities. Note (a), (c) and (e) are based on all data whereas (b), (d) and (f) do not consider data from eight provincial capital cities and Vienna. logPHARM, logged lithium daily defined dose sold per $\mathrm{km}^{2}$; logLITH, logged natural lithium in drinking water. 


\begin{tabular}{|lcr|}
\hline Table 2 & Moran's I results & \\
\hline & Moran's I & \multicolumn{1}{c|}{$P$} \\
\hline SMR, total & 0.414 & $<0.001$ \\
\hline SMR, males & 0.386 & $<0.001$ \\
\hline SMR, females & 0.067 & 0.104 \\
\hline logLITH & 0.539 & $<0.001$ \\
\hline logPHARM & 0.083 & 0.059 \\
\hline $\begin{array}{l}\text { SMR, suicide mortality ratio; logLITH, logged natural lithium in drinking water; } \\
\text { logPHARM, logged lithium daily defined dose sold per km². }\end{array}$ \\
\hline
\end{tabular}

three PCs are used subsequently to adjust for confounding factors in the regressions.

To account for unmodelled residual patterns not explained by the three principal components, spatial Bayesian hierarchical models were estimated. In total, six models were run, namely two each for total, male and female. The modelling strategy comprises the following two models with rising complexity. First, individual effects of $\operatorname{logLITH}$ and $\log$ PHARM on SMRs were tested, while adjusting for a variety of risk factors included as principal components. These models are referred to as 'model 1'. Second, the moderating effect of $\log$ PHARM on $\operatorname{logLITH}$ was tested by means of extending the previous model with a $\operatorname{logLITH}-\log$ PHARM interaction term, representing 'model 2'. The deviance information criterion (DIC) serves for model comparison. A lower DIC score refers to a better fit. All models were set up on 100000 MCMC samples, with 20000 samples discarded during the burn-in period. To incorporate the geography, the models used a neighbourhood matrix based on the five nearest neighbours. Sensitivity runs, with a varying number of nearest neighbours, confirmed the robustness of the model specifications. All DIC scores suggest that models of type 1 rule out type 2 models. Residual independence was confirmed by the Moran's I statistics, supporting the conclusion that spatial autocorrelations were captured appropriately. The posterior distribution of the spatial correlation $(\rho)$ results in a moderate spatial correlation even after controlling for risk factors. Tables 3 and 4 summarise the results of these six models, including the estimated model parameters (i.e. the posterior medians) and the 95\% credible intervals (similar to confidence intervals). It should be noted that if the $95 \%$ credible interval of a variable includes zero, it can be concluded that its effect is of minor relevance.
Model 1 provides considerable evidence that $\operatorname{logLITH}$ has the expected negative influence on suicide. This holds true for the total suicide ratio and for males. This means that higher natural lithium levels decrease the suicide risk. This inverse association is in line with previous studies. ${ }^{3,15}$ Even though the sign of the coefficient for females matches the results for $\mathrm{SMR}_{\mathrm{t}}$ and $S M R_{\mathrm{m}}$, statistical significance was not reached. However, for prescription rates $(\log$ PHARM), a contradictory result in comparison with the bivariate correlations was found. With the exception of the $\mathrm{SMR}_{\mathrm{f}}$ model, showing the anticipated negative sign, the models for $\mathrm{SMR}_{\mathrm{t}}$ and $\mathrm{SMR}_{\mathrm{m}}$ of type 1 have positive $\log \mathrm{PHARM}$ effects, which seem counterintuitive. However, neither for $\mathrm{SMR}_{\mathrm{f}}$ nor for $\mathrm{SMR}_{\mathrm{t}}$ and $\mathrm{SMR}_{\mathrm{m}}$ do the models support that these predictors are of relevance, as the $95 \%$ credible intervals include zero. In a similar study in France, regional prescription rates were negatively associated with male suicide. ${ }^{31}$

Models extended by logLITH-logPHARM moderating effects indicate that interactions were unrelated. This was also reflected in the weaker DIC scores. Furthermore, consideration of a lithium interaction effect in the three extended models 2 absorbed the significance of the originally important contribution of natural lithium, suggesting that $\log$ PHARM does not moderate the $\operatorname{logLITH}$ effect on suicide. Considerable gender differences were noticeable in the principal components controlling for potential confounders. Whereas the first and third principal components were relevant in modelling $\mathrm{SMR}_{\mathrm{t}}$ and $\mathrm{SMR}_{\mathrm{m}}$, this conclusion was not supported by the $\mathrm{SMR}_{\mathrm{f}}$ model. Not surprisingly this confirms the widely recognised fact of gender-specific risk factors as well as a generally lower female suicide risk. ${ }^{4}$ As indicated by regional dummies, evidence exists that suicide rates vary within Austria at a region-wide scale. Compared with southern areas, i.e. Carinthia and Styria, the other regions have a considerably lower suicide risk. This conclusion is valid throughout the models. In addition, small-scale suicide spill-over effects beyond regions were present, efficiently captured by all models. The $\rho$ parameter indicates that complete suicide patterns cannot be explained by confounders alone. This mirrors previous findings ${ }^{6,7}$ emphasising that geography is a crucial factor in explaining suicide patterns.

Finally, the estimated suicide risks, represented as the posterior distributions of the fitted risk patterns of the best performing models (model 1), are illustrated in Fig. 4. Although a general comparison suggests similar risk patterns, a detailed inspection indicates striking differences across space and between

\begin{tabular}{|c|c|c|c|c|c|c|c|c|}
\hline & \multicolumn{4}{|c|}{ Model 1: without interaction effect } & \multicolumn{4}{|c|}{ Model 2: with interaction effect } \\
\hline & \multirow[b]{2}{*}{ Median } & \multicolumn{2}{|c|}{ Credible interval } & \multirow[b]{2}{*}{$P$} & \multirow[b]{2}{*}{ Median } & \multicolumn{2}{|c|}{ Credible interval } & \multirow[b]{2}{*}{$P$} \\
\hline & & $2.5 \%$ & $97.5 \%$ & & & $2.5 \%$ & $97.5 \%$ & \\
\hline Intercept & $0.483^{\mathrm{a}}$ & 0.144 & 0.827 & & 0.441 & -0.212 & 1.113 & \\
\hline IogLITH & $-0.080^{\mathrm{a}}$ & -0.136 & -0.024 & & -0.088 & -0.214 & 0.041 & \\
\hline logPHARM & 0.015 & -0.032 & 0.062 & & 0.029 & -0.176 & 0.226 & \\
\hline $\log L I T H \times \log$ PHARM & & & & & 0.003 & -0.038 & 0.042 & \\
\hline PC1 & $0.035^{\mathrm{a}}$ & 0.004 & 0.065 & & $0.035^{\mathrm{a}}$ & 0.004 & 0.065 & \\
\hline PC2 & 0.022 & -0.009 & 0.052 & & 0.021 & -0.009 & 0.052 & \\
\hline PC3 & $0.052^{\mathrm{a}}$ & 0.018 & 0.085 & & $0.052^{\mathrm{a}}$ & 0.019 & 0.084 & \\
\hline RegEAST & $-0.188^{\mathrm{a}}$ & -0.322 & -0.063 & & $-0.190^{\mathrm{a}}$ & -0.328 & -0.066 & \\
\hline RegMID & $-0.149^{\mathrm{a}}$ & -0.248 & -0.042 & & $-0.149^{\mathrm{a}}$ & -0.250 & -0.039 & \\
\hline RegWEST & $-0.147^{a}$ & -0.250 & -0.038 & & $-0.147^{\mathrm{a}}$ & -0.247 & -0.036 & \\
\hline$\rho$ & $0.370^{\mathrm{a}}$ & 0.040 & 0.860 & & $0.390^{\mathrm{a}}$ & 0.050 & 0.870 & \\
\hline $\mathrm{DIC}$ & -190 & & & & -186 & & & \\
\hline Moran's / errors & -0.019 & & & 0.537 & -0.013 & & & 0.488 \\
\hline
\end{tabular}




\begin{tabular}{|c|c|c|c|c|c|c|c|c|}
\hline & \multicolumn{4}{|c|}{ Model 1: without interaction effect } & \multicolumn{4}{|c|}{ Model 2: with interaction effect } \\
\hline & \multirow[b]{2}{*}{ Median } & \multicolumn{2}{|c|}{ Credible interval } & \multirow[b]{2}{*}{$P$} & \multirow[b]{2}{*}{ Median } & \multicolumn{2}{|c|}{ Credible interval } & \multirow[b]{2}{*}{$P$} \\
\hline & & $2.5 \%$ & $97.5 \%$ & & & $2.5 \%$ & $97.5 \%$ & \\
\hline \multicolumn{9}{|l|}{ Males } \\
\hline Intercept & $0.416^{a}$ & 0.024 & 0.826 & & 0.674 & -0.089 & 1.472 & \\
\hline $\log L I T H$ & $-0.092^{\mathrm{a}}$ & -0.157 & -0.026 & & -0.039 & -0.188 & 0.118 & \\
\hline logPHARM & 0.026 & -0.029 & 0.082 & & -0.065 & -0.311 & 0.168 & \\
\hline $\log L I T H \times \log P H A R M$ & & & & & -0.019 & -0.068 & 0.028 & \\
\hline PC1 & $0.049^{a}$ & 0.012 & 0.084 & & $0.050^{a}$ & 0.015 & 0.086 & \\
\hline PC2 & 0.017 & -0.020 & 0.053 & & 0.016 & -0.022 & 0.052 & \\
\hline PC3 & $0.051^{a}$ & 0.012 & 0.090 & & $0.051^{a}$ & 0.012 & 0.089 & \\
\hline RegEAST & $-0.221^{a}$ & -0.361 & -0.086 & & $-0.223^{\mathrm{a}}$ & -0.368 & -0.086 & \\
\hline RegMID & $-0.161^{a}$ & -0.273 & -0.041 & & $-0.166^{\mathrm{a}}$ & -0.277 & -0.042 & \\
\hline RegWEST & -0.119 & -0.235 & 0.001 & & -0.118 & -0.232 & 0.000 & \\
\hline$\rho$ & $0.360^{a}$ & 0.030 & 0.860 & & $0.370^{a}$ & 0.030 & 0.880 & \\
\hline DIC & -146 & & & & -130 & & & \\
\hline Moran's / errors & -0.016 & & & 0.517 & -0.020 & & & 0.530 \\
\hline \multicolumn{9}{|l|}{ Females } \\
\hline Intercept & 0.613 & -0.009 & 1.254 & & -0.110 & -1.360 & 1.156 & \\
\hline logLITH & -0.044 & -0.148 & 0.059 & & -0.191 & -0.432 & 0.052 & \\
\hline IogPHARM & -0.006 & -0.097 & 0.080 & & 0.243 & -0.145 & 0.635 & \\
\hline $\log \mathrm{LITH} \times \log \mathrm{PHARM}$ & & & & & 0.051 & -0.026 & 0.129 & \\
\hline PC1 & -0.009 & -0.064 & 0.045 & & -0.011 & -0.066 & 0.043 & \\
\hline PC2 & 0.029 & -0.031 & 0.090 & & 0.031 & -0.029 & 0.092 & \\
\hline PC3 & 0.060 & -0.003 & 0.122 & & 0.061 & -0.002 & 0.124 & \\
\hline RegEAST & -0.066 & -0.264 & 0.132 & & -0.068 & -0.266 & 0.128 & \\
\hline RegMID & -0.106 & -0.273 & 0.064 & & -0.102 & -0.275 & 0.068 & \\
\hline RegWEST & $-0.245^{\mathrm{a}}$ & -0.421 & -0.075 & & $-0.248^{a}$ & -0.417 & -0.076 & \\
\hline$\rho$ & $0.350^{\mathrm{a}}$ & 0.010 & 0.880 & & $0.370^{a}$ & 0.020 & 0.890 & \\
\hline DIC & -19 & & & & -13 & & & \\
\hline Moran's / errors & -0.119 & & & 0.977 & -0.107 & & & 0.962 \\
\hline
\end{tabular}

genders. This impression is also confirmed by a correlation analysis between risk surfaces. A high correlation exists between the total risk and the risk for males with a Spearman's $\rho$ of 0.970 $(P<0.001)$. However, the total risk is lower when correlated with the female risk $(\rho=0.730, P<0.001)$ and the correlation between male and female is even lower $(\rho=0.610)$, although highly significant $(P<0.001)$. It is apparent that suicide risk varies significantly across Austria. All risk surfaces show a rough trend of elevated risks from the north to the south with a more pronounced overall risk in the federal state of Styria, which has risk values above 1 . Independently of this risk pattern, the Styrian district Voitsberg has, in particular, a distinctly elevated suicide risk. For that district the total risk is 1.142 , which is even more pronounced for males (1.144). The north-eastern areas, including parts of Lower Austria, Vienna and northern Burgenland, have a reduced risk. For example, the total risk ranges from 0.476 to approximately 0.700 . Remarkably, the estimated female risk surface has a comparable pattern but the magnitude of the risk does not exceed the critical value of 1 across the study area.

\section{Discussion}

\section{Main findings}

The objective of this study was to test the hypothesis whether pharmaceutical lithium prescriptions accumulating in groundwater together with natural lithium in drinking water jointly affect suicide mortality. Utilising spatial Bayesian hierarchical models we looked for this potential co-association in 99 districts in Austria. Although correlation analyses confirm that both natural lithium in drinking water and prescription rates are inversely associated with suicide and both lithium sources are weakly and positively associated, these conclusions are reversed, as soon as risk factors are adjusted for. This may also explain why prior studies ${ }^{16,20,21}$ using a bivariate research design have reported non-significant results regarding suicide and natural lithium levels. More importantly, the models in our study do not support the lithium prescription hypothesis. Both, for total and gender-specific suicide mortalities no evidence was found that lithium-based pharmaceuticals either directly or via accumulation in drinking water diminish the risk of death by suicide. Neither the individual effects nor the lithium interaction effect turned out to be significant when adjusted for confounders, suggesting that lithium in drinking water does not seem to originate from lithium-based pharmaceutical medications (i.e. Quilonorm, Neurolepsin) used in psychiatric treatments. This conclusion is independent of SMR stratification and consistent throughout the models providing additional support that suicide and lithium levels in drinking water are not a function of lithium prescription rates across Austria. However, natural lithium levels in drinking water still provide an explanation for the spatial distribution of suicide mortality. For both the total suicide rates and the male-specific rates the suicide-protective property of natural lithium is confirmed, with a stronger negative effect for males. This outcome corroborates previous studies ${ }^{3,14,15}$ and emphasises the validity of the natural lithium hypothesis stating that lithium in drinking water likely originates from natural sources.

Our results also verify that suicide risk varies considerably across Austria, as indicated by the regional dummy variables that were consistently significant, even after carefully modelling suicide 

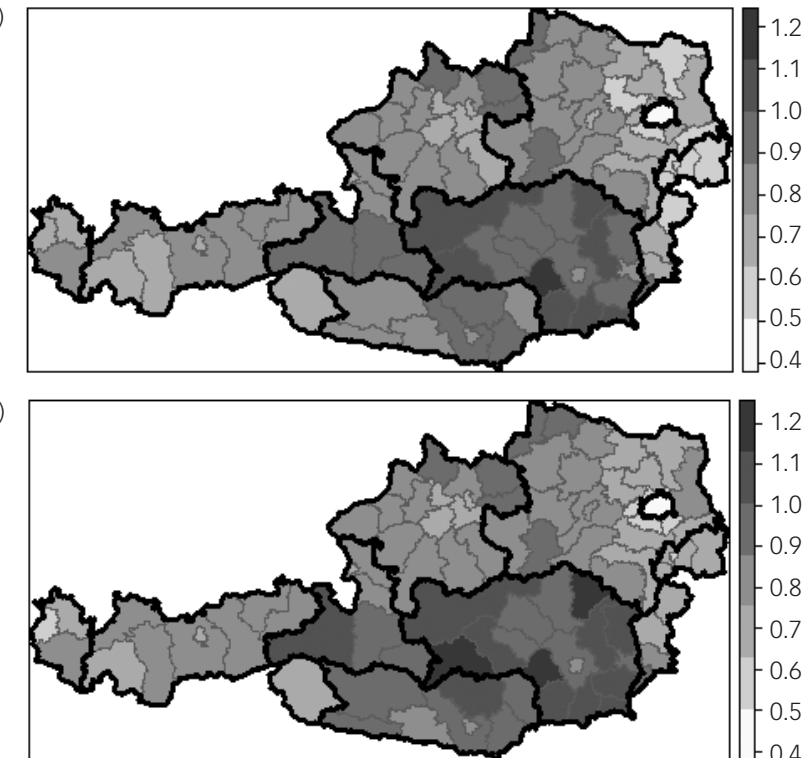

(c)

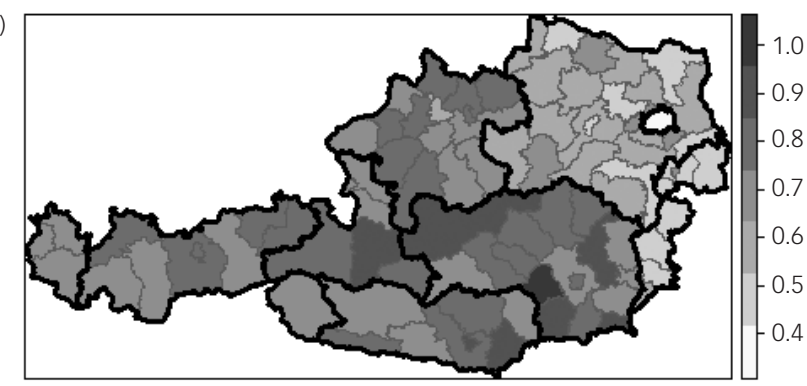

Fig. 4 Estimated suicide risk.

Suicide mortality ratios (a) total, (b) males and (c) females. Dark grey lines delimit federal states; light grey lines define sanitation district boundaries. spill-over effects from adjacent districts through a spatial Bayesian model formulation. This supports findings from Hungary that showed that suicides are geographically agglomerated. ${ }^{7}$ This also supports recent efforts towards a spatial turn in health science, where spatially explicit modelling approaches are receiving increasing attention. ${ }^{6,32}$ Compared with women, men are at greater risk of dying by suicide; ${ }^{1}$ this risk is evidently increased in the southern regions of Austria. In contrast, lowest risk exists in the north-east of Austria. This implies that suicide prevention strategies and programmes must reflect gender dissimilarities and local circumstances in risk patterns by providing specific localised health intervention strategies, such as reducing suicide risk factors and improving protective factors related to mental health well-being.

\section{Strengths and limitations}

By extending previous ecological lithium-suicide studies with the additional of a rational alternative lithium source, namely lithium prescription rates, our study adds to the literature. Besides controlling for a rich set of socioeconomic risk factors and confounding effects of access to health services, a key strength of the study is the larger sample size (of 99 districts) compared with most previous studies. Another major strength is the statistically sound analysis design, explicitly accounting for local suicide spill-over effects while simultaneously controlling for regional heterogeneity in suicide risk. However, some limitations can be identified. In contrast to cohort studies on an individual level, ${ }^{33}$ the present models are cross-sectional and based on ecological regression. Thus, the district-related findings do not allow us to draw conclusions about individuals. Even though the utilised risk and protective factors successfully absorb confounding effects, a few variables could be further stratified by age and/or gender, which might have an impact on the model. Finally, lithium intake via nutrition is not taken into account, which might also contribute to daily lithium exposure. ${ }^{17}$

Despite these reservations, the statistically significant inversely related association between lithium in drinking water and suicide support the validity of the natural lithium hypothesis. No evidence for lithium-based pharmaceutical medication effects on suicide could be confirmed, either as a direct effect or as a moderating effect via natural lithium in drinking water. It is likely, that in comparison with other psychopharmacological prescriptions such as antidepressants, ${ }^{34}$ lithium prescriptions have only a minimal relieving effect on the nations burden of mental disease. Therefore spatial analysis of antidepressant prescriptions on suicide rates, such as that presented by Gibbons et al, ${ }^{35}$ should be considered for further research.

Marco Helbich, PhD, Department of Human Geography and Spatial Planning Utrecht University, Utrecht, The Netherlands; Michael Leitner, PhD, Department of Geography and Anthropology, Louisiana State University, Baton Rouge, Louisiana, USA; Nestor D. Kapusta, PhD, Department for Psychoanalysis and Psychotherapy, Medical University of Vienna, Vienna, Austria

Correspondence: Marco Helbich, Department of Human Geography and Spatial Planning, Utrecht University, Heidelberglaan 2, 3584 CS, Utrecht, The Netherlands. Email: m.helbich@uu.nl

First received 17 Jun 2014, final revision 31 Oct 2014, accepted 2 Nov 2014

\section{Funding}

This research was funded by the Austrian Science Fund (FWF) by grant P25474-B24 and through the Doctoral College GIScience (DK W 1237-N23).

\section{Acknowledgements}

We thank Jakob Klein for his support in data preparation and thank IMS Health, the worldwide information and service leader for the pharmaceutical and health industry, for the data on lithium prescriptions.

\section{References}

1 Hawton K, van Heeringen K. Suicide. Lancet 2009; 373: 1372-81.

2 OECD. Health at a Glance: Europe 2012. OECD Publishing, 2012.

3 Kapusta ND, Mossaheb N, Etzersdorfer E, Hlavin G, Thau K, Willeit M, et al. Lithium in drinking water and suicide mortality. Br J Psychiatry 2011; 198: $346-50$.

4 Hawton K, Casanas C, Haw C, Saunders K. Risk factors for suicide in individuals with depression: a systematic review. J Affect Disord 2013; 147 $17-28$.

5 Helbich M, Leitner M, Kapusta N. Geospatial examination of lithium in drinking water and suicide mortality. Int J Health Geogr 2012; 11: 19.

6 Gunnell D, Wheeler B, Chang S-S, Thomas B, Sterne J, Dorling D. Changes in the geography of suicide in young men: England and Wales 1981. J Epidemiol Community Health 2012; 66: 536-43.

7 Balint L, Dome P, Daroczid G, Gonda X, Rihmer Z. Investigation of the marked and long-standing spatial inhomogeneity of the Hungarian suicide rate: a spatial regression approach. J Affect Disord 2014; 155; 180-5.

8 Harris EC, Barraclough B. Suicide as an outcome for mental disorders A meta-analysis. Br J Psychiatry 1997; 170: 205-28.

9 Müller-Oerlinghausen B, Lewitzka U. Lithium reduces pathological aggression and suicidality: a mini-review. Neuropsychobiology 2010; 62: 43-9.

10 Bschor T. Lithium in the treatment of major depressive disorder. Drugs 2014; 74: 855-62.

11 Cipriani A, Pretty $\mathrm{H}$, Hawton $\mathrm{K}$, Geddes J. Lithium in the prevention of suicidal behavior and all-cause mortality in patients with mood 
disorders: a systematic review of randomized trials. Am J Psychiatry 2005; 162: 1805-19.

12 Baldessarini R, Tondo L, Davis $\mathrm{P}$, Pompili M, Goodwin F, Hennen J. Decreased risk of suicides and attempts during long-term lithium treatment: a meta-analytic review. Bipolar Disord 2006; 8: 625-39.

13 Kessing L, Søndergård L, Kvist $K$, Andersen P. Suicide risk in patients treated with lithium. Arch Gen Psychiatry 2005; 62: 860-6.

14 Schrauzer G, Shrestha K, 1990, Lithium in drinking water and the incidences of crimes, suicides, and arrests related to drug addictions. Biol Trace Elem Res 1990; 25: 105-13.

15 Blüml V, Regier MD, Hlavin G, Rockett IRH, König F, Vyssoki B, et al. Lithium in the public water supply and suicide mortality in Texas. J Psychiatr Res 2013; 47: 407-11

16 Ohgami H, Terao T, Shiotsuki I, Ishii N, Iwata N. Lithium levels in drinking water and risk of suicide. Br J Psychiatry 2009; 194: 464-5.

17 Chandra $\mathrm{P}, \mathrm{Babu}$ G. Lithium in drinking water and food, and risk of suicide. Br J Psychiatry 2009; 195: 271.

18 Huthwaite MA, Stanley J. Lithium in drinking water. Br J Psychiatry 2010; 196: 159.

19 Sugawara N, Yasui-Furukori N, Ishii N, Iwata N, T Terao. Lithium in tap wate and suicide mortality in Japan. Int J Environ Res Public Health 2013; 10: 6044-8.

20 Kabacs N, Memon A, Obinwa T, Stochl J, Perez J. Lithium in drinking water and suicide rates across the East of England. Br J Psychiatry 2011; 198: 406-7

21 Giotakos O, Nisianakis P, Tsouvelas G, Giakalou V-V. Lithium in the public water supply and suicide mortality in Greece. Biol Trace Elem Res 2013; 156 376-9.

22 Concha G, Broberg K, Grandér M, Cardozo A, Palm B, Vahter M. High-level exposure to lithium, boron, cesium, and arsenic via drinking water in the Andes of northern Argentina. Environ Sci Technol 2011; 45: 6575-83.

23 Huang J, Hu H, Lu S, Li Y, Tang F, Lu Y, Wei B. Monitoring and evaluation of antibiotic-resistant bacteria at a municipal wastewater treatment plant in China. Environ Int 2011; 42: 31-6.

24 Rodríguez E, Navarro-Villoslada F, Benito-Peña E, Marazuela M, Moreno-Bondi M. Multiresidue determination of ultratrace levels of fluoroquinolone antimicrobials in drinking and aquaculture water samples by automated online molecularly imprinted solid phase extraction and liquid chromatography. Anal Chem 2011; 83: 2046-55.

25 Kleywegt S, Pileggi V, Yang P, Hao C, Zhao X, Rocks C, et al. Pharmaceuticals, hormones and bisphenol $\mathrm{A}$ in untreated source and finished drinking water in Ontario, Canada - occurrence and treatment efficiency. Sci Total Environ 2011; 409: 1481-8.

26 Fram M, Belitz K. Occurrence and concentrations of pharmaceutical compounds in groundwater used for public drinking-water supply in California. Sci Total Environ 2011; 409: 3409-17.

27 Barnes K, Kolpin D, Furlong E, Zaugg S, Meyer M, Barber L. A national reconnaissance of pharmaceuticals and other organic wastewater contaminants in the United States - I) groundwater. Sci Total Environ 2008; 402: 192-200.

28 Nentwig G. Effects of pharmaceuticals on aquatic invertebrates. Part II: The antidepressant drug fluoxetine. Arch Environ Contam Toxicol 2007; 52: 163-7.

29 World Health Organization. The ICD-10 Classification of Mental and Behavioural Disorders: Clinical Descriptions and Diagnostic Guidelines. WHO, 1992.

30 Lee D. A comparison of conditional autoregressive models used in Bayesian disease mapping. Spat Spatiotemporal Epidemiol 2011; 2: 79-89.

31 Bellanger M, Jourdai A, Batt-Moillo A. Might the decrease in the suicide rates in France be due to regional prevention programmes? Soc Sci Med 2007; 65: 431-41.

32 Helbich M, Blüml V, Leitner M, Kapusta N. Does altitude moderate the impact of lithium on suicide? A spatial analysis of Austria. Geospat Health 2013; 7: 209-18.

33 Termorshuizen F, Wierdsma A, Visser E, Drukker M, Sytema S, Laan W, et al. Psychosis and suicide risk by ethnic origin and history of migration in the Netherlands. Schizophr Res 2012; 138: 268-73.

34 Kapusta ND, Niederkrotenthaler T, Etzersdorfer E, Voracek M, Dervic K, Jandl-Jager $\mathrm{E}$, et al. Influence of psychotherapist density and antidepressant sales on suicide rates. Acta Psychiatr Scand 2009; 119: 236-42.

35 Gibbons RD, Hur K, Bhaumik DK, Mann JJ. The relationship between antidepressant medication use and rate of suicide. Arch Gen Psychiatry 2005; 62: 165-72. 